\title{
A FINITE ELEMENT ANALYSIS OF VISCOELASTICALLY DAMPED SANDWICH PLATES
}

\author{
B.-A. MA \\ Institute of Mechanics, Academia Sinica, Beijing, The People's Republic of China \\ AND \\ J.-F. HE \\ Department of Engineering Mechanics, Tsinghua University, Beijing, \\ The People's Republic of China
}

(Received 30 July 1990, and in revised form 26 November 1990)

\begin{abstract}
A finite element analysis associated with an asymptotic solution method for the harmonic flexural vibration of viscoelastically damped unsymmetrical sandwich plates is given. The element formulation is based on generalization of the discrete Kirchhoff theory (DKT) element formulation. The results obtained with the first order approximation of the asymptotic solution presented here are the same as those obtained by means of the modal strain energy (MSE) method. By taking more terms of the asymptotic solution, with successive calculations and use of the Padé approximants method, accuracy can be improved. The finite element computation has been verified by comparison with an analytical exact solution for rectangular plates with simply supported edges. Results for the same plates with clamped edges are also presented.
\end{abstract}

\section{INTRODUCTION}

Flexural vibrations of damped sandwich plates and structures have been investigated by a number of authors [1-11]. The simplified governing equations and corresponding boundary conditions of flexural vibration of viscoelastically damped unsymmetrical sandwich plates have been given in reference [11]. No bending-extension coupling is included in the simplified governing equations. When a plate is in simple harmonic vibration, the governing differential equations to be solved have complex coefficients. The analytical exact solution and an asymptotic solution were obtained for simply supported rectangular plates in reference [11]. However, an analytical solution of the differential equations can be obtained only in some particular cases. In this paper a finite element analysis associated with an asymptotic solution method for the harmonic vibrations of damped sandwich plates is presented. It is a displacement finite element method, in which triangular elements are used. The element formulation is based on generalization of the discrete Kirchhoff theory (DKT) element formulation presented in references $[12,13]$. The damped sandwich plate is treated initially as fully elastic, and the stiffness and mass matrices of a triangular element are calculated in the usual way. Assembly of the matrices of the whole plate and application of Hamilton's principle lead to the derivation of the matrix equation of motion of the plate. In accordance with the principle of equivalence given in reference [15], the damping is then introduced by replacing the real modulus of the core by its complex counterpart. Therefore, the plate stiffness matrix becomes complex, and a complex matrix equation has to be solved to find the complex frequencies and the corresponding complex 
modes. To obtain an approximate and practical solution and to avoid calculation with complex values, an asymptotic solution of the complex matrix equation is introduced. Calculations have been carried out for two examples and the results verify the effectiveness of the finite element analysis associated with the asymptotic solution method given here.

\section{FINITE ELEMENT FORMULATION}

\subsection{BASIC ASSUMPTIONS AND THE EXPRESSIONS FOR THE ENERGIES}

The basic assumptions for a sandwich plate introduced in reference [11] are also made in this paper: (1) the face-layers are elastic and isotropic and suffer no transverse shear deformation; (2) the core carries transverse shear, but no in-plane stresses; it is linearly viscoelastic and has a complex shear modulus; (3) no slip occurs at the interfaces of the core and face-layers; all points on a normal to the plate move with the same transverse displacement; (4) although the metallic material of the structural layer and the constraining layer may be different, the values of their Poisson ratios are approximately equal; (5) when the sandwich plate is in flexural vibration, the in-plane inertia effects of the plate are ignored and only the transverse inertia effects are considered.

According to these assumptions, one can obtain expressions for the strain energy and the kinetic energy of the plate. They have been given in reference [11]. The strain energy $U$ can be written as the sum of the extension strain energy $U_{e}$ and the bending strain energy $U_{h}$ :

$$
\begin{aligned}
U= & U_{e}+U_{b}=U_{e}+U_{b 1}+U_{b 2}+U_{b 3} \\
= & \frac{1}{2}\left(\gamma_{1}+\gamma_{3}\right) a^{2} \iint_{A}\left[\left(\frac{\partial u_{m}}{\partial \xi}\right)^{2}+2 v \frac{\partial u_{m}}{\partial \xi} \frac{\partial v_{m}}{\partial \eta}+\left(\frac{\partial v_{m}}{\partial \eta}\right)^{2}+\frac{(1-v)}{2}\left(\frac{\partial u_{m}}{\partial \eta}+\frac{\partial v_{m}}{\partial \xi}\right)^{2}\right] \mathrm{d} \xi \mathrm{d} \eta \\
& +\frac{1}{2}\left(D_{1}+D_{3}\right) \iint_{A}\left[\left(\frac{\partial^{2} w}{\partial \xi^{2}}\right)^{2}+2 v \frac{\partial^{2} w}{\partial \xi^{2}} \frac{\partial^{2} w}{\partial \eta^{2}}+\left(\frac{\partial^{2} w}{\partial \eta^{2}}\right)^{2}+2(1-v)\left(\frac{\partial^{2} w}{\partial \xi \partial \eta}\right)^{2}\right] \mathrm{d} \xi \mathrm{d} \eta \\
& +\frac{1}{2}\left(D_{1}+D_{3}\right) Y \iint_{A}\left[\left(\frac{\partial \psi_{\xi}}{\partial \xi}\right)^{2}+2 v \frac{\partial \psi_{\xi}}{\partial \xi} \frac{\partial \psi_{\eta}}{\partial \eta}+\left(\frac{\partial \psi_{\eta}}{\partial \eta}\right)^{2}+\frac{(1-v)}{2}\left(\frac{\partial \psi_{\xi}}{\partial \eta}+\frac{\partial \psi_{\eta}}{\partial \xi}\right)^{2}\right] \mathrm{d} \xi \mathrm{d} \eta \\
& +\frac{1}{2}\left(D_{1}+D_{3}\right) Y g \iint_{A}\left[\left(\psi_{\xi}+\frac{\partial w}{\partial \xi}\right)^{2}+\left(\psi_{\eta}+\frac{\partial w}{\partial \eta}\right)^{2}\right] \mathrm{d} \xi \mathrm{d} \eta
\end{aligned}
$$

Here $A$ is the dimensionless area of the surface of the damped plate and

$$
\begin{gathered}
\gamma_{1}=\frac{E_{1} t_{1}}{1-v^{2}}, \quad \gamma_{2}=G_{2} t_{2}, \quad \gamma_{3}=\frac{E_{3} t_{3}}{1-v^{2}}, \quad D_{1}=\frac{E_{1} t_{1}^{3}}{12\left(1-v^{2}\right)}, \quad D_{3}=\frac{E_{3} t_{3}^{3}}{12\left(1-v^{2}\right)} \\
\xi=x / a, \quad \eta=y / a, \quad u_{m}=U_{m} / a, \quad v_{m}=V_{m} / a, \quad w=W / a \\
U_{m}(x, y, t)=\left[1 /\left(\gamma_{1}+\gamma_{3}\right)\right]\left[\gamma_{1} U_{1}(x, y, t)+\gamma_{3} U_{3}(x, y, t)\right] \\
V_{m}(x, y, t)=\left[1 /\left(\gamma_{1}+\gamma_{3}\right)\right]\left[\gamma_{1} V_{1}(x, y, t)+\gamma_{3} V_{3}(x, y, t)\right] \\
\psi_{\xi}(x, y, t)=(1 / c)\left[U_{1}(x, y, t)-U_{3}(x, y, t)\right] \\
\psi_{\eta}(x, y, t)=(1 / c)\left[V_{1}(x, y, t)-V_{3}(x, y, t)\right] \\
c=t_{2}+(1 / 2)\left(t_{1}+t_{3}\right), \quad Y=\gamma_{1} \gamma_{3} c^{2} /\left(\gamma_{1}+\gamma_{3}\right)\left(D_{1}+D_{3}\right) \\
g=\gamma_{2}\left(\gamma_{1}+\gamma_{3}\right) a^{2} / \gamma_{1} \gamma_{3} t_{2}^{2}
\end{gathered}
$$


where $E_{1}$ and $E_{3}$ are the elastic moduli of the constraining layer (face 1) and the structural layer (face 3), $v$ is their common Poisson ratio, $G_{2}$ is the shear modulus of the viscoelastic core (face 2), which is taken as a real quantity temporarily; and $t_{1}, t_{2}$ and $t_{3}$ are the thicknesses of the three layers respectively. Here $a$ is a characteristic dimension of the plate, e.g., the length of an edge of a rectangular plate; $W(x, y, t)$ is the transverse displacement of the plate in flexural vibration; and $U_{1}, V_{1}$ and $U_{3}, V_{3}$ are the in-plane displacements of the points in the middle planes of face 1 and face 3 respectively. $U_{m}$ and $V_{m}$ may be regarded as the weighted mean in-plane displacements of the unsymmetrical sandwich plate; and $\psi_{\xi}$ and $\psi_{\eta}$ are the rotary angles of a line connecting the two corresponding points at the middle planes of the faces 1 and 3 after bending, $g$ and $Y$ are called the "shear parameter" and the "geometric parameter" respectively. (A list of nomenclature is given in Appendix C.)

The kinetic energy of the plate, $T$, is

$$
T=\frac{1}{2} \rho a^{4} \iint_{A}\left(\frac{\partial w}{\partial t}\right)^{2} \mathrm{~d} \xi \mathrm{d} \eta
$$

where $\rho$ is the mass per unit area of the plate.

When a plate is in simple harmonic flexural vibration, any in-plane rigid-body motions of the plate are suppressed in most cases, so $u_{m}=v_{m}=0$ and then $U_{e}=0$. The forms

$w(\xi, \eta, t)=w(\xi, \eta) \mathrm{e}^{\mathrm{i} \omega t}, \quad \psi_{\xi}(\xi, \eta, t)=\psi_{\xi}(\xi, \eta) \mathrm{e}^{\mathrm{i} \omega t}, \quad \psi_{\eta}(\xi, \eta, t)=\psi_{\eta}(\xi, \eta) \mathrm{e}^{\mathrm{i} \omega t}$,

are introduced. The circular frequency $\omega$ can also be expressed in dimensionless form as

$$
\Omega=\omega \sqrt{\rho a^{4} /\left(D_{1}+D_{3}\right)}
$$

Substituting equation (4) into equations (1) and (3), one can obtain the maximum value of the bending strain energy $U_{b}$ during vibration in the form as in equation (1), and the maximum value of the kinetic energy $T$ during vibration as

$$
T=\frac{1}{2} \rho a^{4} \omega^{2} \iint w^{2} \mathrm{~d} \xi \mathrm{d} \eta=\frac{1}{2}\left(D_{1}+D_{3}\right) \Omega^{2} \iint w^{2} \mathrm{~d} \xi \mathrm{d} \eta
$$

\subsection{DISPLACEMENT MODELS AND STIFFNESS AND MASS MATRICES}

The finite elements used in this paper are triangular plate bending elements with five degrees of freedom at the corner nodes (displacement $w$, rotations $\theta_{\xi}=\partial w / \partial \eta$ and $\theta_{\eta}=$ $-\partial w / \partial \xi$ and rotations $\psi_{\xi}$ and $\psi_{\eta}$ ). The element formulation is based on generalization of the discrete Kirchhoff theory (DKT) element formulation presented in references [12, 13]. There a triangular DKT element with three degrees of freedom at the corner nodes $\left(w, \theta_{\xi}\right.$ and $\theta_{\eta}$ ) was discussed in detail. When an isotropic homogeneous plate with bending stiffness $D$ is in bending, the strain energy can be written as

$$
U=\frac{1}{2} D \iint_{A}\left[\left(\frac{\partial^{2} w}{\partial \xi^{2}}\right)^{2}+2 v \frac{\partial^{2} w}{\partial \xi^{2}} \frac{\partial^{2} w}{\partial \eta^{2}}+\left(\frac{\partial^{2} w}{\partial \eta^{2}}\right)^{2}+2(1-v)\left(\frac{\partial^{2} w}{\partial \xi \partial \eta}\right)^{2}\right] \mathrm{d} \xi \mathrm{d} \eta
$$

Using the DKT elements, one can obtain the global stiffness matrix $\left[k_{D}\right]$ for the element explicitly in the global co-ordinate system, which was presented in reference [13]. It is of order $(9 \times 9)$, and can be divided into nine submatrices of order $(3 \times 3)$ each.

For the triangular plate bending element here, one assumes that $\theta_{\xi}$ and $\theta_{\eta}$ vary quadratically over the element as in the case of the DKT element. Utilizing the expressions of 
equation (26) in reference [12], one can express them as the functions of the area coordinates $\xi_{a}$ and $\eta_{a}$,

$$
\theta_{\xi}=\partial w / \partial \eta=-\left[H_{y}\left(\xi_{a}, \eta_{a}\right)\right]^{\mathrm{T}}\left\{u_{1}\right\}, \quad \theta_{\eta}=-\partial w / \hat{c} \xi=\left[H_{x}\left(\xi_{a} \eta_{a}\right)\right]^{\mathrm{T}}\left\{u_{1}\right\}
$$

where $\left\{u_{1}\right\}$ is composed of part of the nodal degrees of freedom of the element $\left(w_{1}, \theta_{\xi_{1}}, \theta_{\eta 1}, w_{2}, \theta_{\xi_{2}}, \theta_{\eta 2}, w_{3}, \theta_{\xi_{3}}, \theta_{\eta 3}\right)$, and $\left[H_{x}\right]$ and $\left[H_{y}\right]$ are two vectors consisting of nine components and given in Appendix A. However, $\psi_{\xi}$ and $\psi_{\eta}$ are assumed to vary linearly over the element, i.e.,

$$
\psi_{\xi}=\sum_{i=1}^{3} L_{i}\left(\xi_{a} \eta_{a}\right) \psi_{\xi_{i}}, \quad \psi_{\eta}=\sum_{i=1}^{3} L_{i}\left(\xi_{a}, \eta_{a}\right) \psi_{\eta i}
$$

where

$$
L_{1}\left(\xi_{a}, \eta_{a}\right)=1-\xi_{a}-\eta_{a}, \quad L_{2}\left(\xi_{a}, \eta_{a}\right)=\xi_{a}, \quad L_{3}\left(\xi_{a}, \eta_{a}\right)=\eta_{a}
$$

The stiffness matrix of the element here for the analysis of damped sandwich plates is based on the expression of the bending strain energy $U_{b}$ in equation (1). Defining the vector of the nodal degrees of freedom of the element $\{u\}$ as

$$
\{\boldsymbol{u}\}=\left(w_{1}, \theta_{\xi 1}, \theta_{\eta 1}, \psi_{\xi 1}, \psi_{\eta 1}, w_{2}, \theta_{\xi_{2}}, \theta_{\eta 2}, \psi_{\xi_{2}}, \psi_{\eta 2}, w_{3}, \theta_{\xi 3}, \theta_{\eta 3}, \psi_{\xi 3}, \psi_{\eta 3}\right),
$$

one can express $U_{b}$ of an element as

$$
\begin{aligned}
\frac{1}{D_{1}+D_{3}} U_{b} & =\frac{1}{D_{1}+D_{3}}\left(U_{b 1}+U_{b 2}+U_{b 3}\right) \\
& =\frac{1}{2}\{u\}^{\mathrm{T}}\left[k_{1}\right]\{u\}+\frac{1}{2}\{u\}^{\mathrm{T}}\left[k_{2}\right]\{u\}+\frac{1}{2}\{u\}^{\mathrm{T}}\left[k_{3}\right]\{u\}
\end{aligned}
$$

where the area $A$ in the integrations $U_{b 1}, U_{b 2}$ and $U_{b 3}$ in equation (1) is replaced with the dimensionless area $A_{e}$ of the surface of an element. The element stiffness matrix $[k]$ is

$$
[k]=\left[k_{1}\right]+\left[k_{2}\right]+\left[k_{3}\right]
$$

All the matrices $\left[k_{1}\right],\left[k_{2}\right],\left[k_{3}\right]$ and $[k]$ are of order $(15 \times 15)$. Since the expression for $U_{b 1}$ in equation (1) is similar to that for $U$ in equation (7), then $\left[k_{1}\right]$ can be obtained directly from the global stiffness matrix $\left[k_{D}\right]$ of the DKT element presented in reference [13]. At first, the nine submatrices of order $(3 \times 3)$ of $\left[k_{D}\right]$ are each divided by the bending stiffness $D$. Then they are located separately at regions as shown in Figure 1. Finally, some zero elements are added at the rest to form $\left[k_{1}\right]$. The non-zero part of $\left[k_{2}\right]$ can be obtained with a stiffness matrix of order $(6 \times 6)$ given from the displacement model of equation (9). Dividing the matrix into nine submatrices of order $(2 \times 2)$ and locating them separately at the corresponding regions, as shown in Figure 1, one can obtain $\left[k_{2}\right]$ by adding some zero elements. The expression for $\left[k_{3}\right]$ can be obtained from the displacement models of equations (8) and (9) in the usual way. Thereafter, the element stiffness matrix $[k]$ can be constructed in accordance with equation (13). 


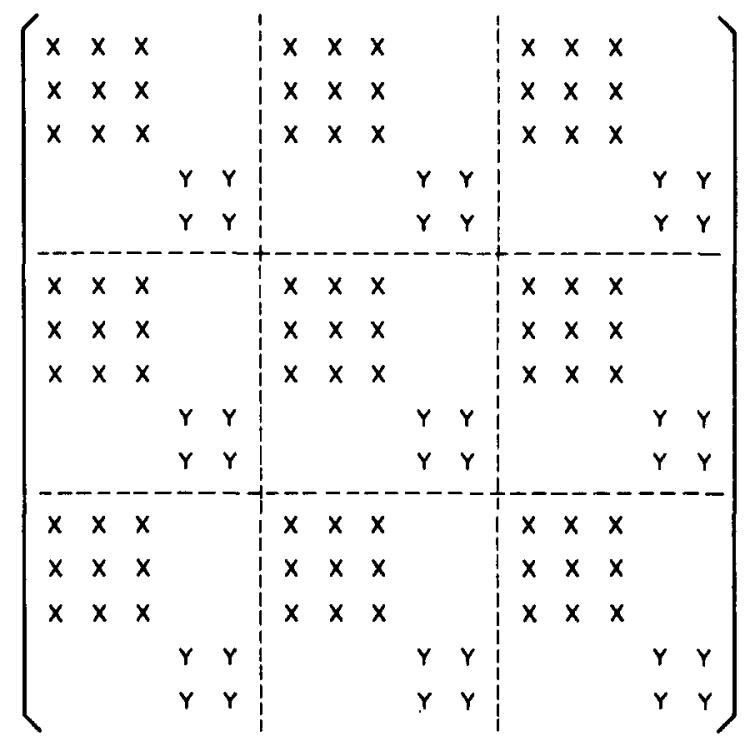

Figure 1. The locations of non-zero elements of $\left[k_{1}\right]$ and $\left[k_{2}\right]$ at an element stiffness matrix $[k]$ of order $(15 \times 15)$. X, elements of $\left[k_{1}\right] ; \mathrm{Y}$, elements of $\left[k_{2}\right]$.

A diagonal lumped mass matrix of the element is employed. One can express the kinetic energy of the element $T$ as follows:

$$
\frac{1}{D_{1}+D_{3}} T=\frac{1}{2} \Omega^{2}\{u\}^{\mathrm{T}}[m]\{u\}
$$

The vector of the diagonal elements of the diagonal mass matrix is given as

$$
\left(A_{e} \mid 3\right)(1,0,0,0,0,1,0,0,0,0,1,0,0,0,0) .
$$

Assembling all the element stiffness and mass matrices of the whole plate and applying Hamilton's principle, one obtains the resulting equation for the damped sandwich plate in simple harmonic vibration as

$$
\left(\left[K_{1}\right]+\left[K_{2}\right]+\left[K_{3}\right]\right)\{q\}-\Omega^{2}[M]\{q\}=[K]\{q\}-\Omega^{2}[M]\{q\}=\{0\},
$$

where $[K]$ and $[M]$ are the stiffness and mass matrices of the whole plate and $\{q\}$ and $\{0\}$ are the displacement vector and zero vector.

However, as the plate is assumed to vibrate harmonically and the core is a layer of viscoelastic damping material, the shear modulus $G_{2}$ of the core in equation (2) has to be replaced by the complex modulus $G_{2}(1+\mathrm{i} \beta)$; here $\beta$ is the loss factor of the viscoelastic material. Then the "shear parameter" $g$ must also be replaced by $g(1+\mathrm{i} \beta)$. The dimensionless frequency $\Omega^{2}$ must also be replaced correspondingly by the complex frequency $\Omega^{2}\left(1+\mathrm{i} \eta^{*}\right)$, the physical significance of which has been discussed in reference [14]. Here the quantity $\eta^{*}$ is called the modal loss factor of the plate. The above changes are justified by the principle of equivalence presented in reference [13]. Therefore, equation (16) becomes instead

$$
\begin{aligned}
\left(\left[K_{1}\right]\right. & \left.+\left[K_{2}\right]+(1+\mathrm{i} \beta)\left[K_{3}\right]\right)\{q\}-\Omega^{2}\left(1+\mathrm{i} \eta^{*}\right)[M]\{q\} \\
& =\left([K]+\mathrm{i} \beta\left[K_{3}\right]\right)\{q\}-\Omega^{2}\left(1+\mathrm{i} \eta^{*}\right)[M]\{q\}=\{0\}
\end{aligned}
$$


This is a complex eigenvalue equation. To investigate flexural vibration of a damped sandwich plate, one must first know the boundary conditions of the plate, which have been discussed in reference [11]. In accordance with different boundary conditions along an edge one has to prescribe some degrees of freedom at the nodes of elements on the edge. For instance, on a simply supported edge (I) $\xi=0$, the displacement $w$ and the rotations $\theta_{\xi}$ and $\psi_{\eta}$ at each node have to be prescribed as zero. On a clamped edge, all the nodal degrees of freedom $w, \theta_{\xi}, \theta_{\eta}, \psi_{\xi}$ and $\psi_{\eta}$ at each node have to be prescribed as zero. Then one can solve equation (17) to find the natural frequencies $\Omega$, the modal loss factors $\eta^{*}$, and the corresponding complex modes $\{q\}$.

\section{THE ASYMPTOTIC SOLUTION OF THE COMPLEX EIGENVALUE EQUATION}

To obtain an approximate and practical solution and to avoid calculation with complex values, an asymptotic solution to the eigenvalue equation (17) with $\mu=\mathrm{i} \beta$ as a complex parameter can be introduced. The same procedure has been used in reference [16] to find the loss factors of a sandwich cantilever. Later, the asymptotic solution to the governing differential equations of damped sandwich plates has been discussed in reference [11]. Here one first expands the solution of equation (17) in a power series:

$$
\begin{gathered}
\{q\}=\{q\}_{0}+\mu\{q\}_{1}+\mu^{2}\{q\}_{2}+\mu^{3}\{q\}_{3}+\mu^{4}\{q\}_{4}+\mu^{5}\{q\}_{5}+\cdots, \\
\Omega^{2}=\Omega_{0}^{2}+\mu^{2} \Omega_{2}^{2}+\mu^{4} \Omega_{4}^{2}+\cdots, \quad \mathrm{i} \eta^{*}=\mu \eta_{1}^{*}+\mu^{3} \eta_{3}^{*}+\mu^{5} \eta_{5}^{*}+\cdots .
\end{gathered}
$$

Substituting equations (18) into equation (17) gives the successive equations that the asymptotic solution must satisfy:

$$
\begin{gathered}
{[K]\{q\}_{0}-\Omega_{0}^{2}[M]\{q\}_{0}=\{0\},} \\
{[K]\{q\}_{1}-\Omega_{0}^{2}[M]\{q\}_{1}=-\left[K_{3}\right]\{q\}_{0}+\Omega_{0}^{2} \eta_{1}^{*}[M]\{q\}_{0},} \\
{[K]\{q\}_{2}-\Omega_{0}^{2}[M]\{q\}_{2}=-\left[K_{3}\right]\{q\}_{1}+\Omega_{0}^{2} \eta_{1}^{*}[M]\{q\}_{1}+\Omega_{2}^{2}[M]\{q\}_{0},} \\
{[K]\{q\}_{3}-\Omega_{0}^{2}[M]\{q\}_{3}=-\left[K_{3}\right]\{q\}_{2}+\Omega_{0}^{2}\left(\eta_{1}^{*}[M]\{q\}_{2}+\eta_{3}^{*}[M]\{q\}_{0}\right)} \\
+\Omega_{2}^{2}\left(\eta_{1}^{*}[M]\{q\}_{0}+[M]\{q\}_{1}\right), \\
{[K]\{q\}_{4}-\Omega_{0}^{2}[M]\{q\}_{4}=-\left[K_{3}\right]\{q\}_{3}+\Omega_{0}^{2}\left(\eta_{1}^{*}[M]\{q\}_{3}+\eta_{3}^{*}[M]\{q\}_{1}\right)} \\
+\Omega_{2}^{2}\left(\eta_{1}^{*}[M]\{q\}_{1}+[M]\{q\}_{2}\right)+\Omega_{4}^{2}[M]\{q\}_{0}, \\
{[K]\{q\}_{5}-\Omega_{0}^{2}[M]\{q\}_{5}=-\left[K_{3}\right]\{q\}_{4}+\Omega_{0}^{2}\left(\eta_{1}^{*}[M]\{q\}_{4}+\eta_{3}^{*}[M]\{q\}_{2}+\eta{ }_{5}^{*}[M]\{q\}_{0}\right)} \\
+\Omega_{2}^{2}\left(\eta_{1}^{*}[M]\{q\}_{2}+\eta_{3}^{*}[M]\{q\}_{0}+[M]\{q\}_{3}\right. \\
+\Omega_{4}^{2}\left(\eta_{1}^{*}[M]\{q\}_{0}+[M]\{q\}_{1}\right),
\end{gathered}
$$

According to various homogeneous boundary conditions of the plate, one finds that boundary conditions which the successive terms $\{q\}_{i}(i=0,1,2, \ldots)$ must satisfy are the same in form. Therefore, from equation (19) and the boundary conditions with respect to $\{q\}_{0}$ one can solve first a real eigenvalue problem and obtain all the eigenvalues $\Omega_{0}^{2}$ and corresponding modes $\{q\}_{0}$. The modes can be normalized as follows.

The normalization of the complex modes can be stated as

$$
\{q\}^{\mathrm{T}}[M]\{q\}=1 .
$$


Substituting the first of equations (18) into equation (25), one can obtain

$$
\begin{gathered}
\{q\}_{0}^{\mathrm{T}}[M]\{q\}_{0}=1, \quad 2\{q\}_{0}^{\mathrm{T}}[M]\{q\}_{1}=0, \\
2\{q\}_{0}^{\mathrm{T}}[M]\{q\}_{2}+\{q\}_{1}^{\mathrm{T}}[M]\{q\}_{1}=0, \quad 2\{q\}_{0}^{\mathrm{T}}[M]\{q\}_{3}+2\{q\}_{1}^{\mathrm{T}}[M]\{q\}_{2}=0, \\
2\{q\}_{0}^{\mathrm{T}}[M]\{q\}_{4}+2\{q\}_{1}^{\mathrm{T}}[M]\{q\}_{3}+\{q\}_{2}^{\mathrm{T}}[M]\{q\}_{2}=0, \\
2\{q\}_{0}^{\mathrm{T}}[M]\{q\}_{5}+2\{q\}_{1}^{\mathrm{T}}[M]\{q\}_{4}+2\{q\}_{2}^{\mathrm{T}}[M]\{q\}_{3}=0, \\
\cdots=0,
\end{gathered}
$$

successively. In accordance with equation (26) one can normalize the real mode $\{q\}_{0}$. Multiplying equation (19) by $\{q\}_{0}^{\mathrm{T}}$, one obtains

$$
\Omega_{0}^{2}=\{q\}_{0}^{\mathbf{T}}[K]\{q\}_{0} .
$$

The expression on the right side of equation (32) is proportional to the strain energy of the plate.

Next one can solve equation (20). First the value of $\eta_{1}^{*}$ must be determined. Multiplying equation (20) by $\{q\}_{0}^{\mathrm{T}}$ and considering equation (26) and

$$
\{q\}_{0}^{\mathrm{T}}\left([K]\{q\}_{1}-\Omega_{0}^{2}[M]\{q\}_{1}\right)=\{q\}_{1}^{\mathrm{T}}\left([K]\{q\}_{0}-\Omega_{0}^{2}[M]\{q\}_{0}\right)=0,
$$

one obtains

$$
\eta_{1}^{*}=\left(1 / \Omega_{0}^{2}\right)\{q\}_{0}^{\mathrm{T}}\left[K_{3}\right]\{q\}_{0} .
$$

$\eta_{1}^{*}$ is the fraction of strain energy attributable to the viscoelastic core when the damped plate deforms in the mode $\{q\}_{0}$. If less accuracy is acceptable, all the first terms of each expression in equations (18) can be regarded as an approximate solution, which are the same as the results obtained by means of the modal strain energy (MSE) method suggested in references [9] and [10]. Therefore, MSE can be considered to be the first order approximation of the asymptotic solution presented in this paper.

To improve accuracy, one must calculate successive terms of the asymptotic solution. Substituting the values of $\Omega_{0}^{2}, \eta_{1}^{*}$ and $\{q\}_{0}$ into equation (20), one obtains a system of linear simultaneous equations which has infinite solutions for $\{q\}_{1}$. At first any particular solution $\{q\}_{1 p}$ can be obtained; then the general solution for $\{q\}_{1}$ may be written in the form

$$
\{q\}_{1}=\{q\}_{1 p}+C_{1}\{q\}_{0},
$$

where $C_{1}$ is an undetermined constant. The value of $C_{1}$ can be given by means of the normalization condition equation (27) as

$$
C_{1}=-\{q\}_{0}^{\mathrm{T}}[M]\{q\}_{1 p} .
$$

Then the values of $\{q\}_{1}$ can be determined completely.

Thereafter one solves equation (21). Multiplying equation (21) by $\{q\}_{0}^{\mathrm{T}}$, one can obtain

$$
\Omega_{2}^{2}=\{q\}_{0}^{\mathrm{T}}\left[K_{3}\right]\{q\}_{1} .
$$

Substituting the values of $\Omega_{0}^{2}, \eta_{1}^{*}, \Omega_{2}^{2},\{q\}_{0}$ and $\{q\}_{1}$ into equation (21), one can obtain the unique solution for $\{q\}_{2}$ as

$$
\{q\}_{2}=\{q\}_{2 p}+C_{2}\{q\}_{0}
$$


where the value of $C_{2}$ is given in accordance with the normalization condition equation (28) as

$$
\left.C_{2}=-\{q\} 0[M]\{q\}_{2 p}^{\mathrm{T}}-\frac{1}{2}\{q\}\right\}_{1}^{\mathrm{T}}[M]\{q\}_{1}^{\prime},
$$

Through calculations similar to those previously, the solutions of the successive equations (22) -(24) can be obtained as follows:

$$
\begin{aligned}
& \eta_{3}^{*}=-\eta_{1}^{*}\left(\{q\}_{0}^{\mathrm{T}}[M]\{q\}_{2}+\Omega_{2}^{2} / \Omega_{0}^{2}\right)+\left(1 / \Omega_{0}^{2}\right)\{q\}_{0}^{\mathrm{T}}\left[K_{3}\right]\{q\}_{2}, \\
&\{q\}_{3}=\{q\}_{3 p}+C_{3}\{q\}_{0}, \quad C_{3}=-\{q\}_{0}^{\mathrm{T}}[M]\{q\}_{3 p}-\{q\}_{1}^{\mathrm{T}}[M]\{q\}_{2},\left\{\Omega_{4}^{2}=\{q\}_{0}^{\mathrm{T}}\left[K_{3}\right]\{q\}_{3}-\Omega_{0}^{2} \eta_{1}^{*}\{q\}_{0}^{\mathrm{T}}[M]\{q\}_{3}-\Omega_{2}^{2}\{q\}_{0}^{\mathrm{T}}[M]\{q\}_{2}^{\prime},\right. \\
& \Omega_{4}=\{q\}_{4 p}+C_{4}\{q\}_{0}, \\
& C_{4}=-\{q\}_{0}^{\mathrm{T}}[M]\{q\}_{4 p}-\{q\}_{1}^{\mathrm{T}}[M]\{q\}_{3}-\frac{1}{2}\{q\}_{2}^{\mathrm{T}}[M]\{q\}_{2}, \\
& \eta_{5}^{*}=-\eta_{1}^{*}\left(\{q\}_{0}^{\mathrm{T}}[M]\{q\}_{4}+\left(\Omega_{2}^{2} / \Omega_{0}^{2}\right)\{q\}_{0}^{\mathrm{T}}[M]\{q\}_{2}+\Omega_{4}^{2} / \Omega_{0}^{2}\right) \\
&-\eta_{3}^{*}\left(\{q\}_{0}^{\mathrm{T}}[M]\{q\}_{2}+\Omega_{2}^{2} / \Omega_{0}^{2}\right) \\
&+\left(1 / \Omega_{0}^{2}\right)\left(\{q\}_{0}^{\mathrm{T}}\left[K_{3}\right]\{q\}_{4}-\Omega_{2}^{2}\{q\}_{0}^{\mathrm{T}}[M]\{q\}_{3}\right), \\
& \quad\{q\}_{5}=\{q\}_{5 p}+C_{5}\{q\}_{0}, \\
& C_{5}=-\{q\}_{0}^{\mathrm{T}}[M]\{q\}_{5 p}-\{q\}_{1}^{\mathrm{T}}[M]\{q\}_{4}-\{q\}_{2}^{\mathrm{T}}[M]\{q\}_{3}, \\
& \cdots=\cdots .
\end{aligned}
$$

If the calculation is not to be continued, one is left with the expressions for the asymptotic solution given explicitly in equations (18), without the residual terms indicated by $(.$. on their right sides. The last two of equations (18) may be rewritten as

$$
\begin{aligned}
& \Omega^{2}=\Omega_{0}^{2}\left[1+\beta^{2}\left(\frac{\Omega_{4}^{2}}{\Omega_{2}^{2}}-\frac{\Omega_{2}^{2}}{\Omega_{0}^{2}}\right)\right]\left(1+\beta^{2} \frac{\Omega_{4}^{2}}{\Omega_{2}^{2}}\right), \\
& \eta^{*}=\eta_{1}^{*} \beta\left[1+\beta^{2}\left(\frac{\eta_{5}^{*}}{\eta_{3}^{*}}-\frac{\eta_{3}^{*}}{\eta_{1}^{*}}\right)\right]\left(1+\beta^{2} \frac{\eta_{5}^{*}}{\eta_{3}^{*}}\right) .
\end{aligned}
$$

Equations (49) and (50) can be obtained by using the Padé approximants method (see Appendix B). By calculating with equations (49) and (50), one can often obtain better results.

\section{EXAMPLES}

\subsection{EXAMPLE I}

Consider a rectangular viscoelastically damped sandwich plate with simple support (I) along all edges. Its length is $a$ and its width is $(1 / 1 \cdot 2) a$. Introduce a dimensionless coordinate system, $0, \xi, \eta$ and let the edges of the plate be $\xi=0,1$ and $\eta=0,1 / 1 \cdot 2$. Two hundred triangular elements with 121 nodes are used to form a regular mesh, as shown in 


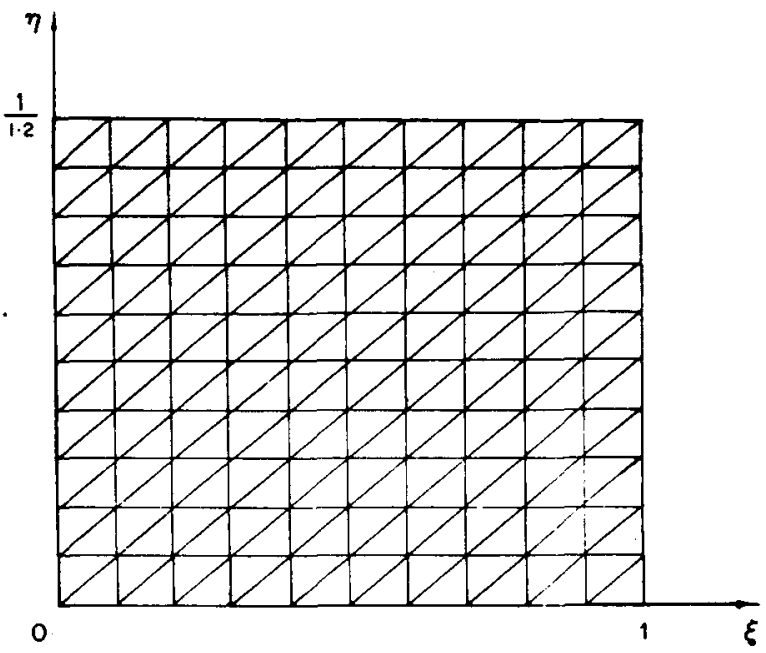

Figure 2. A rectangular damped sandwich plate and the 200 triangular elements used to form the mesh.

Figure 2. The analytical exact solution for the natural frequencies, modes and modal loss factors of such plates has been given in reference [11]. Numerical computation by the finite element method associated with the asymptotic solution method was carried out for the plate with $\beta=1.0$ and $Y=3.5$. The first four natural flexural vibrations of the plate were calculated. Let $m$ and $n$ be the semi-wavenumbers of the natural mode $w$ along the directions of $\xi$ and $\eta$. The semi-wavenumbers $(m, n)$ of the first four natural modes $w$ are $(1,1),(2,1),(1,2)$ and $(2,2)$ respectively. Define $\alpha_{m n}=m^{2} \pi^{2}+(1 \cdot 2)^{2} n^{2} \pi^{2}$. In Figures 3 and 4 are shown the numerical results for the first four modes of the plate. In Figure 3 the curves representing the variation of $\Omega^{2} / \Omega_{e x}^{2}$ with the variable $g / \alpha_{m n}$ are given, where the values of $\Omega^{2}$ have been calculated according to $\Omega_{0}^{2}$ or equation (49). $\Omega_{e x}^{2}$ is the analytical exact solution given in reference [11]. The values of $\Omega^{2}$ calculated according to equation (49) approximate to the analytical exact values $\Omega_{e x}^{2}$ much better than the values

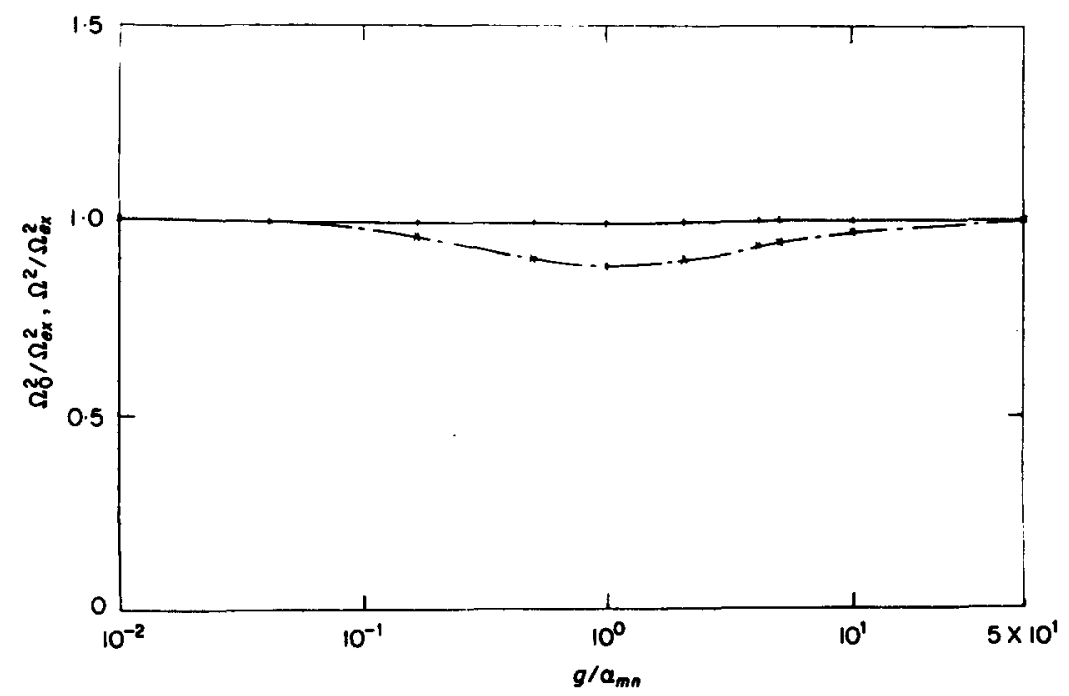

Figure 3. Ratio of the numerical asymptotic solutions of natural frequencies to the analytical exact ones $v s$. the variable $g / \alpha_{m n} .---, \Omega_{0}^{2} / \Omega_{e x}^{2} ;-, \Omega^{2} / \Omega_{e x}^{2}$. 


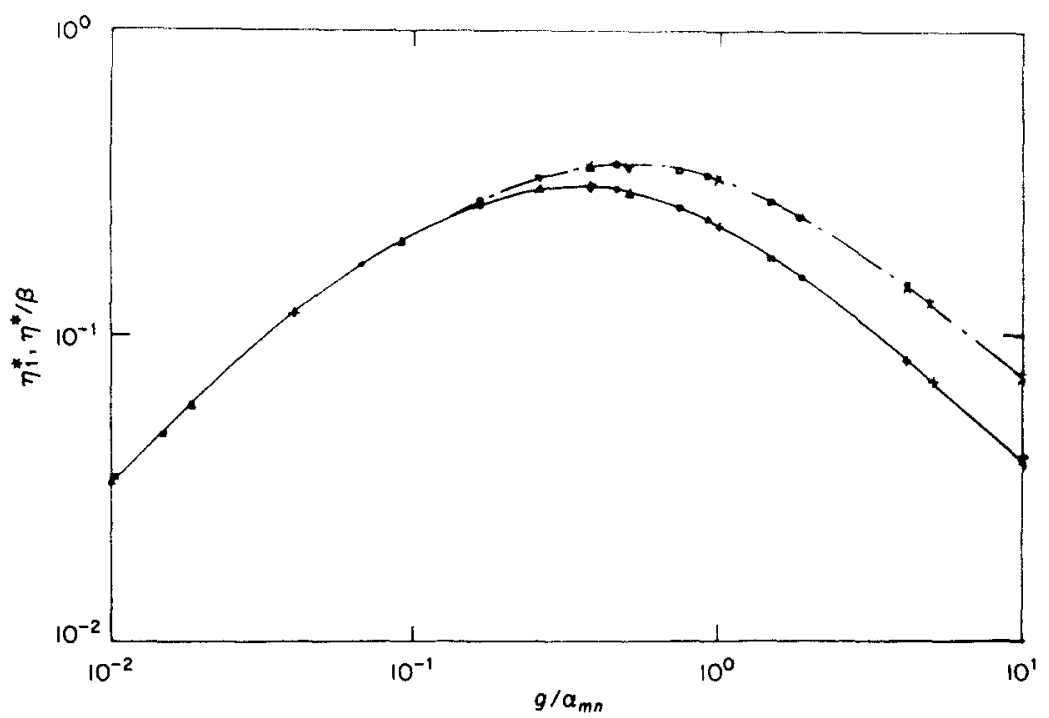

Figure 4. Ratio of $\eta^{*}$ to $\beta$ vs. the variable $g / \alpha_{m n} . Y=3 \cdot 5, \beta=1 .-\cdots, \eta_{1}^{*} ;--, \eta^{*} / \beta ; \times,+,(m, n)=$ $(1,1) ; \bigcirc, \bigcirc,(m, n)=(2,1) ; \square, \mathbf{\square},(m, n)=(1,2) ; \nabla, \Delta,(m, n)=(2,2)$.

$\Omega_{0}^{2}$, which are equivalent to the result obtained by the MSE method. In Figure 4 is shown $\eta^{*} / \beta$ as a function of the variable $g / \alpha_{m n}$, where the values of $\eta^{*}$ can be calculated according to $\eta_{1}^{*} \beta$ or equation (50). The solid curve represents the exact variation $\eta^{*} / \beta$ with the variable $g / \alpha_{m n}$, which was given in reference [11]. The numerical results of $\eta^{*} /$ $\beta$ calculated according to equation (50) approximate the solid curve very well. In Table 1 the values of $\Omega_{0}^{2}, \Omega_{2}^{2}, \Omega_{4}^{2}$ and $\Omega^{2}$ calculated according to equation (49) for corresponding values of $g / \alpha_{11}$ to be $0.5,1$ and 5 are given. The exact values $\Omega_{e x}^{2}$ are also given for comparison. In Table 2 the values of $\eta_{1}^{*}, \eta_{3}^{*}, \eta_{5}^{*}$ and $\eta^{*}$ calculated according to equation (50) and the analytical exact values $\eta_{e x}^{*}$ for the same values of $g / \alpha_{11}$ are given.

To observe the influence of number of elements on the accuracy of the results, the values calculated by employing 72 elements with 49 nodes are also given in Tables 1 and 2 . It

TABLE 1

Dimensionless frequencies of simply supported plates with different values of $g / \alpha_{11}$ $(Y=3 \cdot 5, \beta=1 \cdot 0)$

\begin{tabular}{ccccccc}
\hline$g / \alpha_{11}$ & $\begin{array}{c}\text { Number of } \\
\text { elements }\end{array}$ & $\Omega_{0}^{2}$ & $\Omega_{2}^{2}$ & $\Omega_{4}^{2}$ & $\Omega^{2}$ & $\Omega_{e x}^{2}$ \\
\hline $0 \cdot 5$ & 72 & 1218 & -144 & -16 & 1347 & 1392 \\
& 200 & 1244 & -148 & -16 & 1378 & 1392 \\
1 & 72 & 1545 & -242 & -61 & 1738 & 1798 \\
& 200 & 1580 & -251 & -62 & 1782 & 1798 \\
5 & 72 & 2220 & -230 & -156 & 2358 & 2410 \\
& 200 & 2257 & -235 & -162 & 2396 & 2410 \\
\hline
\end{tabular}

can be seen that convergence is rapid in most cases. From Tables 1 and 2 it is evident that the errors in the values of $\Omega_{0}^{2}$ and $\eta_{1}^{*}$ are somewhat appreciable for these values of $g / \alpha_{11}$ in comparison with the analytical exact values of $\Omega_{e x}^{2}$ and $\eta_{e x}^{*}$, while the values of $\Omega^{2}$ and $\eta^{*}$ are much better. 
TABLE 2

Modal loss factors of simply supported plates with different values of $\mathrm{g} / \alpha_{11}$ $(Y=3 \cdot 5, \beta=1 \cdot 0)$

\begin{tabular}{ccccccc}
\hline $\boldsymbol{g} / \boldsymbol{\alpha}_{11}$ & $\begin{array}{c}\text { Number of } \\
\text { elements }\end{array}$ & $\eta^{*}$ & $\eta^{*}$ & $\eta^{*}$ & $\eta^{*}$ & $\boldsymbol{\eta}_{\text {ex }}^{*}$ \\
\hline $\mathbf{0 . 5}$ & 72 & 0.3572 & 0.0818 & 0.0189 & $\mathbf{0 . 2 9 0 7}$ & $\mathbf{0 . 2 9 1 7}$ \\
& 200 & 0.3597 & 0.0824 & 0.0189 & $\mathbf{0 . 2 9 2 7}$ & $\mathbf{0 . 2 9 1 7}$ \\
\multirow{2}{*}{1} & 72 & 0.3189 & 0.1284 & 0.0525 & 0.2278 & 0.2258 \\
& 200 & 0.3197 & 0.1299 & 0.0529 & $\mathbf{0 . 2 2 7 3}$ & 0.2258 \\
5 & 72 & 0.1356 & 0.0989 & 0.0781 & $\mathbf{0 . 0 8 0 3}$ & $\mathbf{0 . 0 6 9 0}$ \\
& 200 & $\mathbf{0 . 1 2 6 2}$ & 0.0996 & 0.0793 & $\mathbf{0 . 0 7 0 8}$ & $\mathbf{0 . 0 6 9 0}$ \\
\hline
\end{tabular}

\subsection{EXAMPLE 2}

Consider the same rectangular plate as in Example 1 but with all clamped edges. The element mesh is also the same in Figure 2. There is no exact solution of this problem. The first four natural vibrations with semi-wavenumbers of modes $w(1,1),(2,1),(1,2)$ and $(2,2)$ were also calculated. In Figures 5-8 curves showing the variation of $\Omega_{0}^{2} / \Omega^{2}$ with

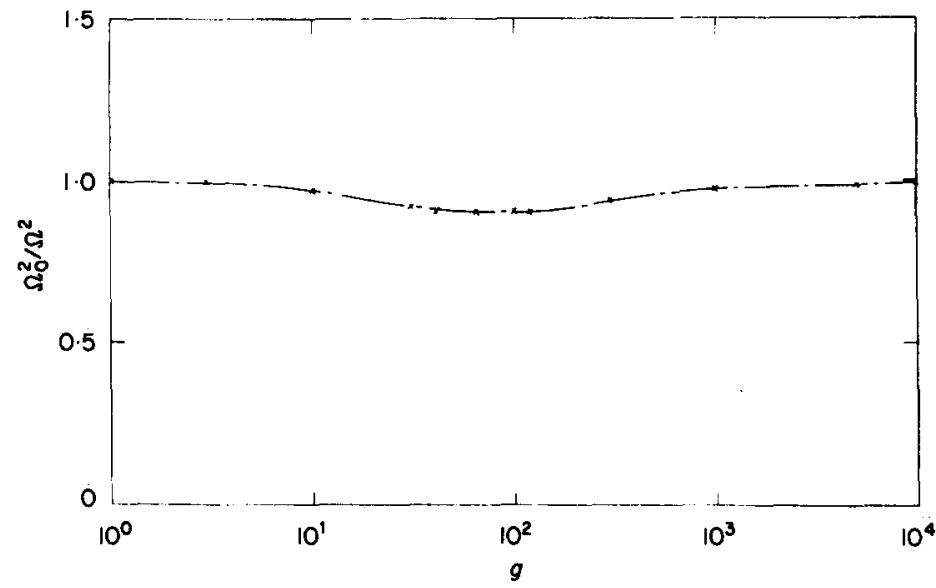

Figure 5. Ratio of $\Omega_{0}^{2}$ to $\Omega^{2}$ vs. the variable $g .(m, n)=(1,1)$.

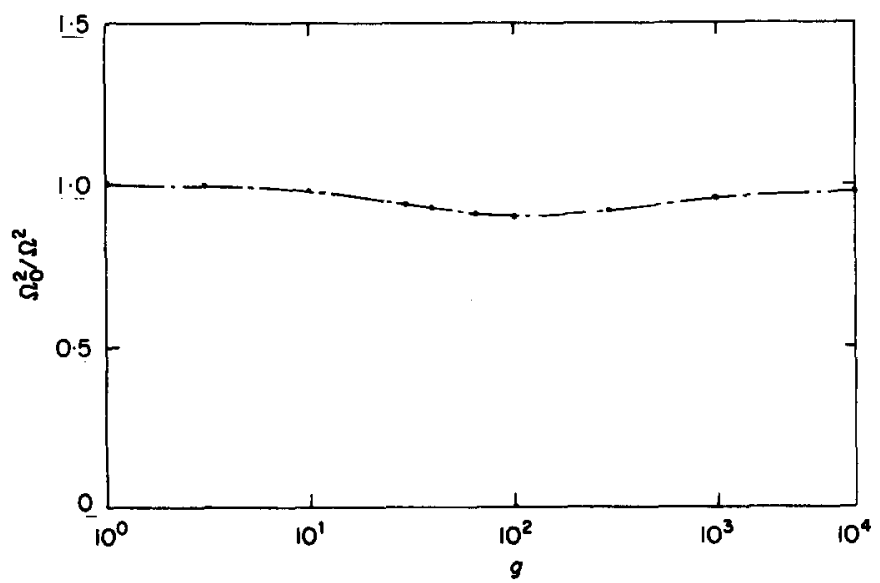

Figure 6. Ratio of $\Omega_{0}^{2}$ to $\Omega^{2}$ vs. the variable $g .(m, n)=(2,1)$. 


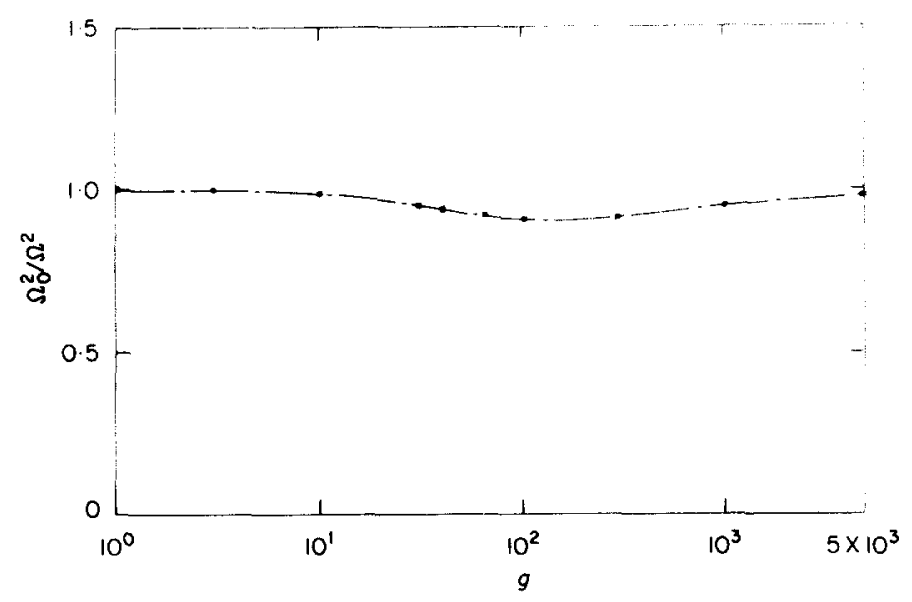

Figure 7. Ratio of $\Omega_{0}^{2}$ to $\Omega^{2}$ vs. the variable $g .(m, n)=(1,2)$.

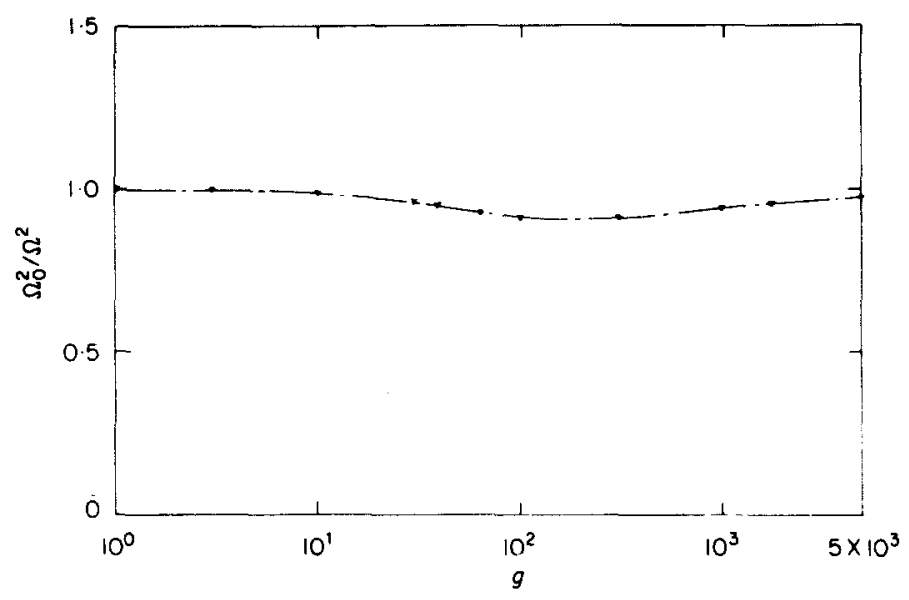

Figure 8. Ratio of $\Omega_{0}^{2}$ to $\Omega^{2}$ vs. the variable $g .(m, n)=(2,2)$.

the variable $g$ for the first four natural vibrations are given, respectively, with the values of $\Omega^{2}$ having been calculated according to equation (49). In Figures $9-12$ is shown $\eta^{*} / \beta$ as a function of the variable $g$, where the values of $\eta^{*}$ were calculated according to $\eta_{1}^{*} \beta$ or equation (50). The results are similar to those in Example 1.

\section{CONCLUSIONS}

In this paper a finite element analysis associated with an asymptotic solution method for the harmonic flexural vibrations of viscoelastically damped sandwich plates has been given. The element formulation is based on generalization of the DKT element formulation. To avoid calculation with complex values, an asymptotic solution of the complex governing matrix equation has been introduced, with the loss factor of the viscoelastic material of the core used as a parameter. As sample examples, calculations have been carried out for rectangular plates with either simply supported or clamped edges. The first four natural frequencies and modal loss factors of the plates are given. If in the asymptotic solution only the first terms of all quantities are adopted, then the result is identical in principle with that as given in accordance with the modal strain energy (MSE) method. 


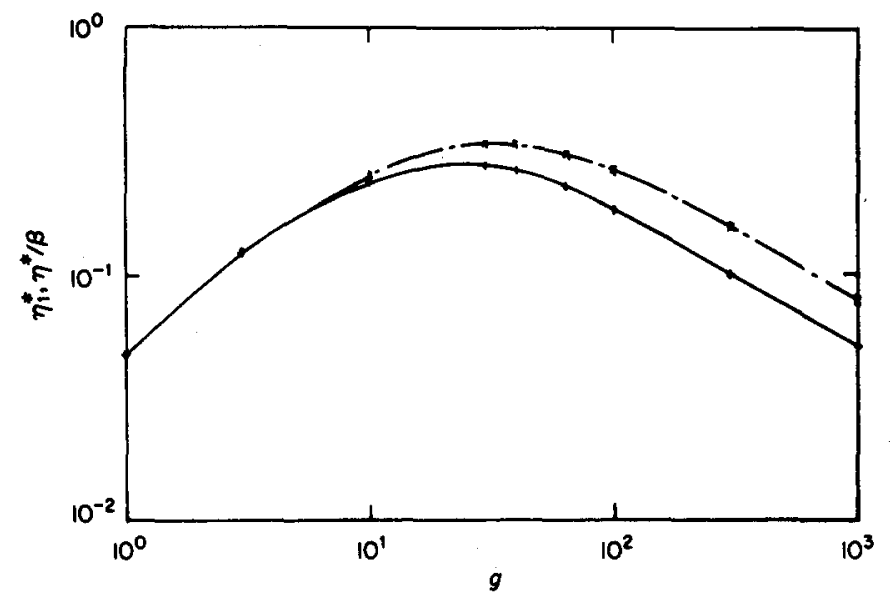

Figure 9. Ratio of $\eta^{*}$ to $\beta$ us. the variable $g .(m, n)=(1,1) .-\cdots, \eta_{1}^{*} ;-,, \eta^{*} / \beta$.

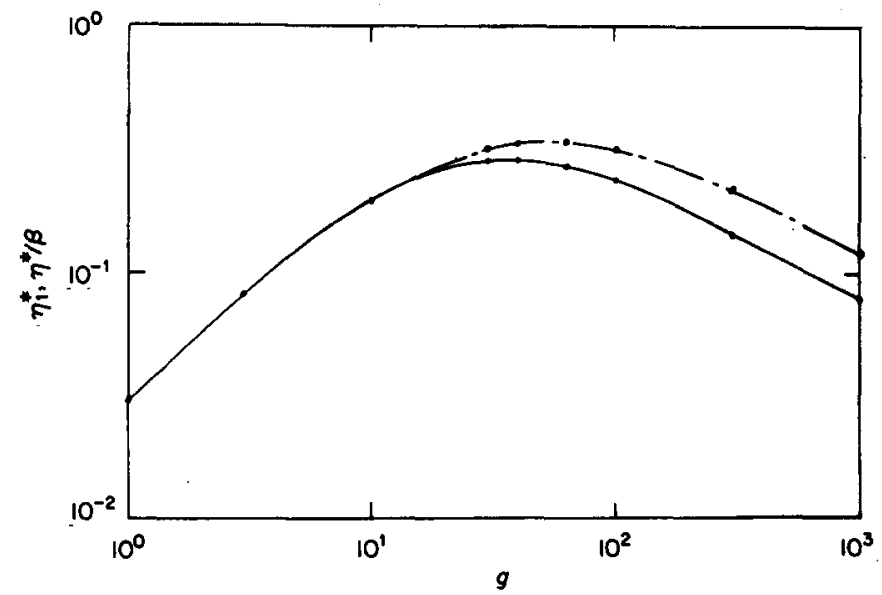

Figure 10. Ratio of $\eta^{*}$ to $\beta$ vs. the variable $g .(m, n)=(2,1) .-\cdots, \eta_{1}^{*} ;-, \eta^{*} / \beta$.

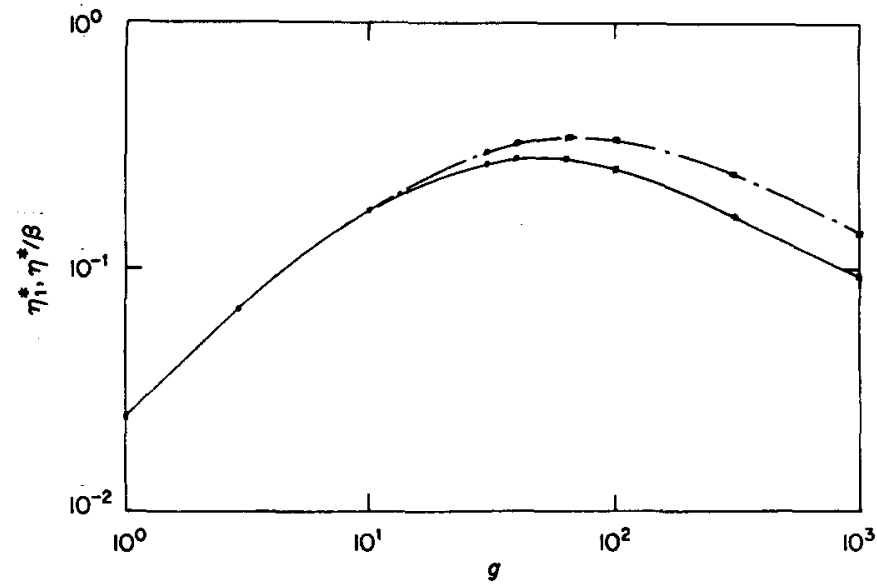

Figure 11. Ratio of $\eta^{*}$ to $\beta$ vs. the variable $g .(m, n)=(1,2) .-\cdots, \eta_{1}^{*} ;-,, \eta^{*} / \beta$. 


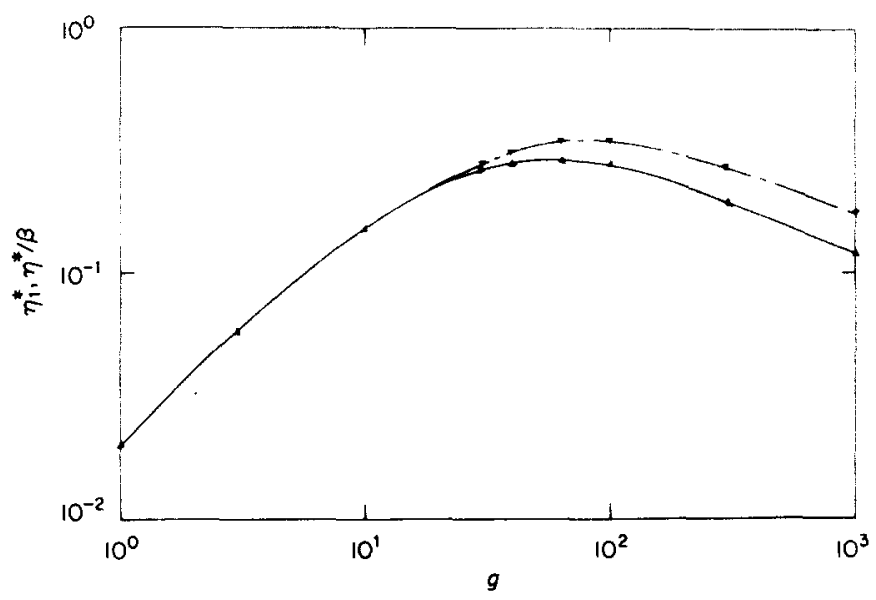

Figure 12. Ratio of $\eta^{*}$ to $\beta$ vs. the variable $g .(m, n)=(2,2),-\cdot-, \eta_{1}^{*} ;-\cdots, \eta^{*} / \beta$.

However, the results of the examples indicate that the errors in the values of the natural frequencies and modal loss factors are somewhat appreciable in a certain range of the value of the "shear parameter" $g$. By taking more terms of the asymptotic solution, with successive calculations and use of the Padé approximants method, accuracy can be improved. For rectangular plates with simply supported edges (Example 1), the numerical results approximate to the corresponding analytical exact values very well. This fact verifies the reliability of the finite element analysis associated with the asymptotic solution method presented here. With the character of the free flexural vibrations of a plate thus calculated, then it is possible to analyze further the response of the plate to various types of dynamic loads to provide a reliable basis for design.

\section{ACKNOWLEDGMENT}

The support by the National Science Foundation of China is gratefully acknowledged.

\section{REFERENCES}

1. D. J. MeAd 1972 Journal of Sound and Vibration 24, 275-295. The damping properties of elastically supported sandwich plates.

2. Y. V. K. S. RAO and B. C. NAKRA 1973 Archives of Mechanics 25(2), 213-225. Theory of vibratory bending of unsymmetrical sandwich plates.

3. Y. V. K. S. RAO and B. C. NAKRA 1974 Journal of Sound and Vibration 34, 309-326. Vibrations of unsymmetrical sandwich beams and plates with viscoelastic cores.

4. E. IOANNides and P. GrootenhuIs 1979 Journal of Sound and Vibration 67, 203-218. A finite element analysis of the harmonic response of damped three-layer plates.

5. D. J. MEAD 1980 Proceedings of the Applied Research Laboratory Seminar on Vibration Control 80-10. Vibration control with sandwich beams and plates.

6. S. MARkus and T. NANASI 1981 Journal of Sound and Vibration 76, 421-441. Significance of in-plane inertia forces in the vibration analysis of three-layered circular plates.

7. Y. P. LU and G. C. Everstine 1980 Journal of Sound and Vibration 69, 199-205. More on finite element modeling of damped composite systems.

8. M. L. SONI and F. K. BOGNER 1982 American Institute of Aeronautics and Astronautics Journal 20(5), 700-707. Finite element vibration analysis of damped structures.

9. C. D. JOHNSON and D. A. KIENHOLz 1982 American Institute of Aeronautics and Astronautics Journal 20(9), 1284-1290. Finite element prediction of damping in structures with constrained viscoelastic layers. 
10. D. A. Kienholz, C. D. Johnson and J. C. Parekh 1983 Proceedings of 24th AIAA/ASME/ ASCE/AHS Structures, Structural Dynamics and Materials Conference 83-0904. Design methods for viscoelastically damped plates.

11. J.-F. HE and B.-A. MA 1988 Journal of Sound and Vibration 126, 37-47. Analysis of flexural vibration of viscoelastically damped sandwich plates.

12. J.-L. BAtoz, K.-J. BAthe and L.-W. Ho 1980 International Journal for Numerical Methods in Engineering 15, 1771-1812. A study of three-node triangular plate bending elements.

13. C. JeyachandRabose, J. Kirkhope and C. Ramesh BABU 1985 International Journal for Numerical Methods in Engineering 21, 1289-1293. An alternative explicit formulation for the DKT plate-bending element.

14. D. J. Mead and S. Markus 1969 Journal of Sound and Vibration 10, 163-175. The forced vibration of a three-layer, damped sandwich beam with arbitrary boundary conditions.

15. M. A. Biot 1955 in IUTAM Madrid Colloquism (R. Grammel, editor), 251-263. Heidelberg: Springer-Verlag. Variational and Lagrangian methods in viscoelasticity.

16. V. Oravsky, S. Markus and O. Simkova 1974 Journal of Sound and Vibration 33, 335-352. A new approximate method of finding the loss factors of a sandwich cantilever.

17. H. Cabannes 1976 Padé Approximants Method and its Applications to Mechanics. Berlin: Springer-Verlag.

\section{APPENDIX A: EXPRESSIONS FOR $\left[H_{x}\left(\xi_{a}, \eta_{a}\right)\right]$ AND $\left[H_{y}\left(\xi_{a}, \eta_{a}\right)\right]$}

The vectors $\left[H_{x}\left(\xi_{a}, \eta_{a}\right)\right]$ and $\left[H_{y}\left(\xi_{a}, \eta_{a}\right)\right]$ were given in reference [12] and are listed here. They are nine-component vectors of shape functions. The expressions for the first three components of them are

$$
\begin{gathered}
H_{x 1}=1 \cdot 5\left(a_{6} N_{6}-a_{5} N_{5}\right), \quad H_{x 2}=b_{5} N_{5}+b_{6} N_{6}, \quad H_{x 3}=N_{1}-c_{5} N_{5}-c_{6} N_{6}, \\
H_{y 1}=1 \cdot 5\left(d_{6} N_{6}-d_{5} N_{5}\right), \quad H_{y 2}=-N_{1}+e_{5} N_{5}+e_{6} N_{6}, \quad H_{y 3}=-H_{x 2},
\end{gathered}
$$

where $N_{i}(i=1, \ldots, 6)$ are

$$
\begin{gathered}
N_{1}=2\left(1-\xi_{a}-\eta_{a}\right)\left(\frac{1}{2}-\xi_{a}-\eta_{a}\right), \quad N_{2}=\xi_{a}\left(2 \xi_{a}-1\right), \quad N_{3}=\eta_{a}\left(2 \eta_{a}-1\right), \\
N_{4}=4 \xi_{a} \eta_{a}, \quad N_{5}=4 \eta_{a}\left(1-\xi_{a}-\eta_{a}\right), \quad N_{6}=4 \xi_{a}\left(1-\xi_{a}-\eta_{a}\right) .
\end{gathered}
$$

The components $H_{x 4}, H_{x 5}, H_{x 6}, H_{y 4}, H_{y 5}$ and $H_{y 6}$ are obtained from the expressions (A1) and (A2) by replacing $N_{1}$ by $N_{2}$ and indices 6 and 5 by 4 and 6 , respectively. The components $H_{x 7}, H_{x 8}, H_{x 9}, H_{y 7}, H_{y 8}$ and $H_{y 9}$ are obtained by replacing $N_{1}$ by $N_{3}$ and indices 6 and 5 by 5 and 4 , respectively. Also,

$$
\begin{gathered}
a_{k}=-\xi_{i j} / l_{i j}^{2}, \quad b_{k}=3 \xi_{i j} \eta_{i j} /\left(4 l_{i j}^{2}\right), \quad c_{k}=\left(\xi_{i j}^{2}-2 \eta_{i j}^{2}\right) /\left(4 l_{i j}^{2}\right), \quad d_{k}=-\eta_{i j} / l_{i j}^{2}, \\
e_{k}=\left(\eta_{i j}^{2}-2 \xi_{i j}^{2}\right) /\left(4 l_{i j}^{2}\right), \quad \xi_{i j}=\xi_{i}-\xi_{j}, \quad \eta_{i j}=\eta_{i}-\eta_{j}, \quad l_{i j}=\left(\xi_{i j}^{2}+\eta_{i j}^{2}\right)^{1 / 2},
\end{gathered}
$$

where the index $k=4,5$ and 6 for indices $i j=23,31$ and 12 respectively. Here $J_{i}$ and $\eta_{i}$ $(i=1,2,3)$ are the values of the nodal Cartesian co-ordinates of the triangular element.

\section{APPENDIX B: PADÉ APPROXIMANTS METHOD}

For several years, the Padé approximants method has become important in engineering. The underlying principle of Padé's method is as follows. Given a power series

$$
f(x)=\sum_{k=0}^{\infty} c_{k} x^{k}
$$


Padé proposed finding the closest approximation to the sum by defining a rational fraction $P_{m}(x) / Q_{n}(x)$, with

$$
P_{m}(x)=\sum_{k=0}^{m} a_{k} x^{k}, \quad Q_{n}(x)=1+\sum_{k=1}^{n} b_{k} x^{k}
$$

in which the numerator and the denominator are polynomials of degrees $m$ and $n$ respectively. These polynomials $P_{m}(x)$ and $Q_{n}(x)$ are determined from the equation

$$
f(x)-P_{m}(x) / Q_{n}(x)=O\left(x^{m+n+1}\right) .
$$

This equation leads to $m+n+1$ linear simultaneous equations from which the unknown $m+n+1$ values $a_{k}$ and $b_{k}\left(b_{0}=1\right)$ can be determined.

For example, the square of the natural frequencies of a damped sandwich plate $\Omega^{2}$ is given as a power series in equation (18) in this paper. The expression can be rewritten as

$$
\Omega^{2}=\Omega_{0}^{2}-\beta^{2} \Omega_{2}^{2}+\beta^{4} \Omega_{4}^{2}-\cdots .
$$

One can find a rational fraction $P_{1}\left(\beta^{2}\right) / Q_{1}\left(\beta^{2}\right)$, with

$$
P_{1}\left(\beta^{2}\right)=a_{0}+a_{1} \beta^{2}, \quad Q_{1}\left(\beta^{2}\right)=1+b_{1} \beta^{2},
$$

as the closest approximation to the value of $\Omega^{2}$. In accordance with equation (B3) one can obtain the linear simultaneous equations

$$
\Omega_{0}^{2}=a_{0}, \quad-\Omega_{2}^{2}+\Omega_{0}^{2} b_{1}=a_{1}, \quad \Omega_{4}^{2}-\Omega_{2}^{2} b_{1}=0 .
$$

These equations lead to

$$
a_{0}=\Omega_{0}^{2}, \quad a_{1}=\Omega_{0}^{2}\left(\frac{\Omega_{4}^{2}}{\Omega_{2}^{2}}-\frac{\Omega_{2}^{2}}{\Omega_{0}^{2}}\right), \quad b_{1}=\frac{\Omega_{4}^{2}}{\Omega_{2}^{2}} .
$$

Then one obtains equation (49) in this paper. Equation (50) is obtained similarly.

The mathematical theory and applications of the Padé approximants method can be found, e.g., in reference [17].

\section{APPENDIX C: NOMENCLATURE}

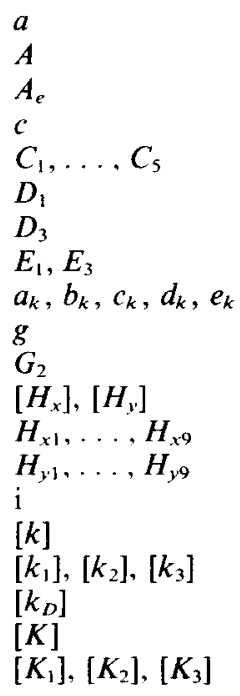

characteristic dimension of the plate dimensionless area of the surface of the plate dimensionless area of an element distance between the middle planes of face-layer 1 and face-layer 3 constants bending rigidity of the constraining layer (face-layer 1) bending rigidity of the structural layer (face-layer 3 ) Young's moduli of the face-layers 1 and 3 coefficients in the expressions for $H_{x 1}, \ldots, H_{x 9}$ shear parameter shear modulus of the viscoelastic core (layer 2) nine component vectors of shape functions components of vector $\left[H_{x}\right]$ components of vector $\left[H_{y}\right]$ $\sqrt{-1}$ element stiffness matrix three parts of an element stiffness matrix element stiffness matrix of a DKT element stiffness matrix of the whole plate three parts of stiffness matrix of the whole plate assembled with three parts 


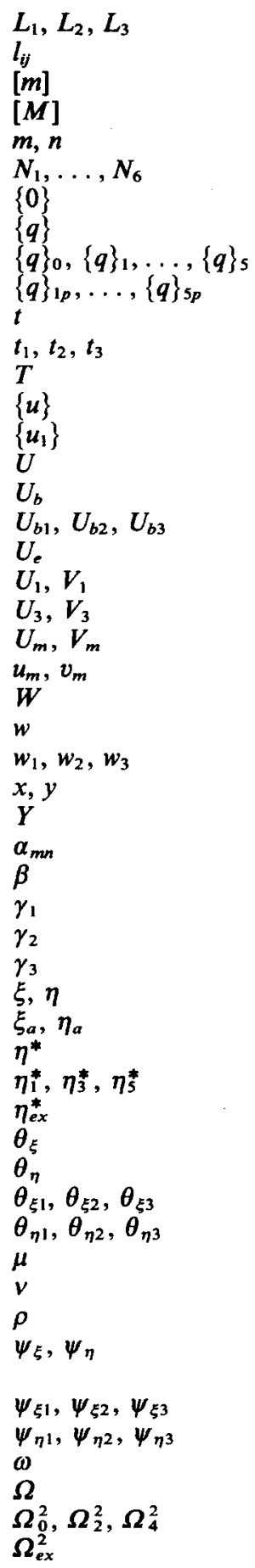

of element stiffness matrices

area co-ordinate functions of a triangular element

$\sqrt{\xi_{i j}^{2}+\eta_{i j}^{2}}$, coefficient in the expressions of $a_{k}, b_{k}, \ldots, e_{k}$

diagonal element mass matrix

mass matrix of the whole plate

semi-wavenumbers

shape functions for DKT element

zero vector

displacement vector

successive vectors in power series for $\{q\}$

particular solutions for $\{q\}_{1}, \ldots,\{q\}_{5}$

time

thicknesses of the layers 1,2 and 3

kinetic energy of the plate

15-component vector of the nodal degrees of freedom of an element

9-component vector of part of the nodal degrees of freedom of the element strain energy of a sandwich (or homogeneous) plate

bending strain energy

three parts of the bending strain energy

extension strain energy

in-plane displacements of the points in the middle plane of face-layer 1

in-plane displacements of the points in the middle plane of face-layer 3

weighted mean in-plane displacements of an unsymmetrical sandwich plate dimensionless weighted mean in-plane displacements

transverse displacement

dimensionless transverse displacement

nodal values of dimensionless transverse displacements

rectangular Cartesian co-ordinates

geometric parameter

$m^{2} \pi^{2}+(1 \cdot 2)^{2} n^{2} \pi^{2}$

loss factor of the viscoelastic material

$E_{1} t_{1} /\left(1-v^{2}\right)$

$G_{2} t_{2}$

$E_{3} t_{3} /\left(1-v^{2}\right)$

dimensionless rectangular Cartesian co-ordinates

area co-ordinates of a triangular element

modal loss factor of the plate

coefficients of successive terms in power series for $\eta^{*}$

analytical exact values of modal loss factor

$\partial w / \partial \eta$, rotations vary over an element

$-\partial w / \partial \xi$, rotations vary over an element

nodal values of $U_{\xi}$

nodal values of $\theta_{\eta}$

$\mathrm{i} \beta$, complex parameter in power series

common Poisson ratio of the face-layers 1 and 3

mass per unit area of the plate

rotary angles of a line connecting the two corresponding points at the middle planes of the face-layers 1 and 3 after bending

nodal values of $\psi_{\xi}$

nodal values of $\psi_{\eta}$

circular frequency

dimensionless circular frequency

coefficients of successive terms in power series for $\Omega^{2}$

analytical exact values of $\Omega^{2}$ 